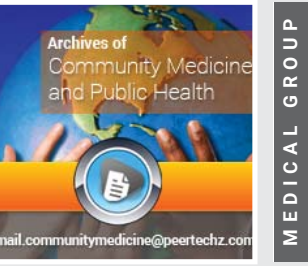

\section{The role of volunteers in the} radiology department of wuhan leishenshan hospital: A mini review from a single study \section{center}

\section{Jinxiang Hu and Ming Deng*}

Department of Radiology, Zhongnan Hospital of Wuhan University, Wuhan University, Wuhan 430071, PR China
Received: 21 July, 2020

Accepted: 28 July, 2020

Published: 30 July, 2020

*Corresponding author: Ming Deng, Department of Radiology, Zhongnan Hospital of Wuhan University, Wuhan University, \#169 Donghu Road, Wuchang District, Wuhan city, Hubei province 430071 , China, Tel: +86-153-9284-1757; Fax: 027-67811731;

E-mail:dr_dengming@whu.edu.com

Keywords: Volunteers work; Hospital management; Radiology department; Humanistic care; COVID-19; SARS-Cov-2

https://www. peertechz.com

Check for updates

\begin{abstract}
This retrospective report, detailed the volunteer work of the radiology department in Wuhan Leishenshan Hospital at the COVID-19 epidemic period. Under the background of the lack of medical staff, we pointed out that the special hospital is not restricted in one pattern, and, which has laid a solid foundation for the effective development of the medical treatment. During the past two months, the volunteers showed strong humanistic care and selfless dedication, which provided manpower guarantee for spreading positive energy and conquering the COVID-19 epidemic as soon as possible. Such a program could serve as a model for common reference throughout the world. The author summarizes the report and pay tribute to the volunteers from all over the world.
\end{abstract}

\section{Introduction}

In late December 2019, many cases of "unknown viral pneumonia" were reported in China and were later determined to be caused by a novel coronavirus, severe acute respiratory syndrome coronavirus 2 (SARS-CoV-2) [1-3]. In the context of the coronavirus disease 2019 (COVID-19) epidemic, the Chinese government built a new designated hospital for diagnosed cases of COVID-19, named Wuhan Leishenshan Hospital, from January 27 and February 7, 2020. Due to the timing of the COVID-19 outbreak, Wuhan's Leishenshan Hospital is world-famous for its rapid construction and high-quality care of COVID-19 patients [4]. From the official, the hospital is composed of 1500 beds and 32 wards, including two Intensive Care Units (ICU). The first ward of the hospital began to admit patients on February 8. Other wards were opened under the concept of "construction, acceptance, training and admission". All 32 wards were initiated on February 20. From February 8 to April 14, 2,011 patients, including 899 patients with severe illness and 179 patients with critical illness, 55\% of patients were over 60 years, were treated.
Studies have shown that CT and X-ray, which are contained in the radiology department, are important imaging modalities for COVID-19, especially in the diagnosis of disease and evaluation of therapeutic effect after treatment $[1,5,6]$. The Radiology Department of Wuhan Leishenshan Hospital is located in the medical image building and is equipped with three high-performance CT scanners, one of which was special equipment for a medical examination for medical staff or workers, the other two CT were designed as examination machines for patients with COVID-19.

The 10-day-old hospital has an isolation ward area of 52,000 square meters and consists of two fishbone-shaped isolation wards for COVID-19. Between the two isolation wards is the patient channel with 200 meters long. In the context of the COVID-19 epidemic, the large area of Wuhan Leishenshan Hospital, more wards, and long patient channels to increase the difficulty for inpatients; besides, because most of the patients with complex critical condition, unfamiliar with the surroundings, and unable to complete the examination independently. Therefore, the accurate guidance of each 
patient is particularly important. In this context, the band of in-patient areas and radiology examination room is necessary [7].

During the outbreak period of the COVID-19 epidemic, especially in Wuhan, the medical staff was in short supply. Therefore, how to obtain volunteers to ensure the patients to complete the CT examination in an orderly and efficient way has become an urgent problem in the imaging department. This article summarizes the role of volunteers in the Department of Radiology of Wuhan Leishenshan Hospital under COVID-19 epidemic, to provide a reference for hospital management and radiologists to cope with the process of imaging examination.

\section{Volunteer selection}

We have very high requirements for the selection of volunteers with the following criteria (1). Because of the CT examination before and after, volunteers may have to carry patients, so we prefer male health volunteers to join (2). Most of the patients in Leishenshan Hospital are elderly (some of them have one more chronic disease) and special group (patients with a mental abnormality, senile dementia, deaf, blind, and pregnant women), volunteers are required to have good communication skills with a humanistic care (3). To minimize the occurrence of emergencies during the CT examination, and to identify changes in patients' behavior or conditions at an early stage, we want to choose people with some nursing experience or medical knowledge background as our volunteers. In the end, eight male volunteers (age range from 25 to 38 years) joined the team, including two university students and two hospital administrators [8].

\section{Volunteer training setting}

The chair of the Radiology Department and the Senior Matron systematically trained the selected volunteers. The training contents include four basic requirements. First, to understand the CT examination process, radiation protection knowledge, and key points for safety. Second, to be familiar with the location of each isolation ward and patient access; Third, be familiar with the working route of medical staff and the layout of cleaning area, semi-polluted area and polluted area of medical image building; Fourth, training the wear and take off the sequence of protective equipment and matters needing attention. Volunteers are required to accomplish all the above assessments and qualified before allowing working.

\section{The main work of Volunteers}

\section{Transporting patients are an important part of the vo- lunteer's day job}

First, to arrange the patients for CT examination safety, the radiology department, and clinical departments to establish the Wechat contact platform. The detail of patients (including the number of patients with severe illness and those who cannot take care of themselves) who need to be examined by CT is published by clinical departments through the platform, and indicate the AIDS (E. G. Ambulance, wheelchair or suction machine) that are required for special patient transport.
To prevent the disorder of patient flow, resulting in poor management of radiology. The Volunteer group precisely controls the patients flow from the isolation ward to the CT examination room.

\section{Spiritual Encouragement and humanistic care}

During the current epidemic, patients infected with the COVID-19 became isolated and isolated from their families, often accompanied by depression. When the volunteers take the patients out of the isolation ward, we encourage volunteers and patients to take the initiative to relieve patients' anxiety and fear. Second, to better communicate special care and positive energy, we encourage volunteers to take photos with patients at appropriate times and to share treatment progress with family and friends to assist patients to boost their confidence and overcome their fear of COVID-19.

\section{Help the patient complete the examination}

Detailed instructions and gentle reminders are posted in patient channels and waiting areas in Leishenshan Hospital. The volunteers explain the process of CT examination according to the instruction picture and instruct the patient to control breathing during the examination, help the patient to take off the accessories, which may affect the examination. In the process of preparation, the volunteers actively note the changes in patients' conditions; record the patients' possible critical risks, and real-time transmission to the hospital information system, prompting the inspectors to prepare in advance. In addition to assisting the technician to complete the whole process, the volunteers also need to do some basic care for special patients, including assisting in patting the back and turning over the body of patients [7].

\section{The division of labor and cooperation of volunteers}

The radiology administrator arranges the volunteers 'daily work in progress based on the number of patients collected on the daily information release platform and the assistance needed to transport patients (such as ambulances, flatbeds, wheelchairs, etc.). We divided the volunteers into two groups, each with three, in the morning and the afternoon two shifts. Each group appointed a rotating team leader, in charge of waiting area patient management and pre-scanning interpretation. Another two volunteers were under the responsibility of the transfer of patients from the ward. Each volunteer equipped with a walkie-talkie, real-time voice communication with the radiologists, ambulance drivers and technicians, so that all the problem timely feedback [9]. Volunteers are relatively fixed, not private changing of the guard. Each volunteer works no more than 4 hours a day, and is supervised and managed by the hospital quality control staff.

Over the past two months, eight volunteers in the radiology department had high-density contact with COVID-19 patients for more than 60 days, and 4,929 CT examinations were completed, reaching a maximum of 245 per day. At present, the mission of Leishenshan Hospital has been fulfilled, and the volunteer work has ended. Happily, the reverse transcriptionpolymerase chain reaction test and SARS-Cov-2 antibody test 
of all volunteers confirmed negative, and volunteers would like to thank contributors to the prevention of the COVID-19 epidemic.

In conclusion, volunteers are essential during the outbreaks period. Due to the participation of volunteers in Leishenshan Hospital, who link the examination and treatment, the CT examination process is more efficient. However, they are just a few of the many volunteers. The author believes that the spirit of volunteerism deserves the respect of people all over the world.

\section{Acknowledgments}

We thank all our colleagues who helped us during the current study. We express the authors'warmly gratitude to the volunteers, who participated in the epidemic prevention of Leishenshan Hospital, and pay high tribute to the spirit of volunteers all over the world.

\section{References}

1. Li L, Qin L, Xu Z, Yin Y, Wang X, et al. (2020) Artificial Intelligence Distinguishes COVID-19 from Community Acquired Pneumonia on Chest CT. Radiology 2020: 200905. Link: https://bit.ly/3hlYzSO
2. Lee EYP, Ng MY, Khong PL (2020) COVID-19 pneumonia: what has CT taught us? Lancet Infect Dis 20: 384-385. Link: https://bit.ly/2CSObLu

3. Deng M (2020) The prevention and management of the coronavirus disease 2019 (COVID-19) outbreak in radiology departments in epidemic areas. Jpn J Radiol 1-6. Link: https://bit.ly/309zAlH

4. Wang $Y Y$, Jin $Y H$, Ren XQ, Li YR, Zhang XC, et al. (2020) Updating the diagnostic criteria of COVID-19 "suspected case" and "confirmed case" is necessary. Mil Med Res 7: 17. Link: https://bit.ly/2P5ho6B

5. Li K, Wu J, Wu F, Guo D, Chen L, et al. (2020) The Clinical and Chest CT Features Associated with Severe and Critical COVID-19 Pneumonia. Invest Radiol. Link: https://bit.ly/2BKXPxi

6. Joob B, Wiwanitkit V (2020) Computed Tomographic Findings in COVID-19. Korean J Radiol 21: 620-622. Link: https://bit.ly/30VDSMK

7. Etkind SN, Bone AE, Lovell N, Cripps RL, Harding R, et al. (2020) The role and response of palliative care and hospice services in epidemics and pandemics: a rapid review to inform practice during the COVID-19 pandemic. J Pain Symptom Manage 60: e31-e40. Link: https://bit.ly/337EYrr

8. Soled D, Goel S, Barry D, Erfani P, Joseph N, et al. (2020) Medical Student Mobilization During A Crisis: Lessons From A COVID-19 Medical Student Response Team. Acad Med. Link: https://bit.ly/3faFiYP

9. National Committee on Covid-19 Epidemiology $\mathrm{MoH}$, Medical Education IRI (2020) Daily Situation Report on Coronavirus disease (COVID-19) in Iran; March 17, 2020. Arch Acad Emerg Med 8: e28.Link: https://bit.ly/39EoOIL
Discover a bigger Impact and Visibility of your article publication with

\section{Peertechz Publications}

Copyright: @ $2020 \mathrm{Hu} \mathrm{J}$, et al. This is an open-access article distributed under the terms of the Creative Commons Attribution License, which permits unrestricted use, distribution, and reproduction in any medium, provided the original author and source are credited. 